\title{
VECTOR-VALUED FUZZY MULTIFUNCTIONS
}

\author{
ISMAT BEG \\ Lahore University of Management Sciences \\ Department of Mathematics \\ 54792 Lahore Cantt., Pakistan
}

(Received October, 1999; Revised February, 2001)

\begin{abstract}
Some of the properties of vector-valued fuzzy multifunctions are studied. The notion of sum fuzzy multifunction, convex hull fuzzy multifunction, close convex hull fuzzy multifunction, and upper demicontinuous are given, and some of the properties of these fuzzy multifunctions are investigated.
\end{abstract}

Key words: Fuzzy Multifunction, Fuzzy Topological Vector Space, Fuzzy Topological Space, Fuzzy Analysis. 46А99.

AMS subject classifications: 46S40，47S40，47H04，04A72，03E72,

\section{Introduction}

In the last three decades, the theory of multifunctions has advanced in a variety of ways. The theory of multifunctions was first codified by Berge [8]. Applications of this theory can be found in economic theory, noncooperative games, artificial intelligence, medicine, and existence of solutions for differential inclusions (see Aubin and Ekeland [2], Klein and Thompson [15], Aubin and Frankowska [3], and the references therein). Recently, Heilpern [11], Butnariu [9], Albrycht and Maltoka [1], Papageorgiou [20], Ozbakir and Aslim [19], Tsiporkova-Hristoskova, De Baets and Kerre [2224], and Beg [4-7] have started the study of fuzzy multifunctions and hemicontinuous fuzzy multifunctions. The aim of this paper is to study properties of vector-valued fuzzy multifunctions. The notion of sum fuzzy multifunction, and upper demicontinuous fuzzy multifunction are given, and some of the properties of these fuzzy multifunctions are investigated.

\section{Preliminaries}

Let $X$ be an arbitrary (nonempty) set. A fuzzy set (in $X$ ) is a function with domain $X$ and values in $[0,1]$. If $A$ is a fuzzy set and $x \in X$, the function $A(x)$ is called the grade of membership of $x$ in $A$. The fuzzy set $A^{c}$, defined by $A^{c}(x)=1-A(x)$, is 
called the complement of $A$. Let $A$ and $B$ be fuzzy sets in $X$. We write $A \subseteq B$ if $A(x) \leq B(x)$ for each $x \in X$. For any family $\left\{A_{i}\right\}_{i \in I}$ of fuzzy sets in $X$, we define:

$$
\left[\bigcap_{i \in I} A_{i}\right](x)=\inf _{i \in I} A_{i}(x)
$$

and

$$
\left[\bigcup_{i \in I} A_{i}\right](x)=\sup _{i \in I} A_{i}(x) .
$$

The family $\tau$ of fuzzy sets in $X$ is called a fuzzy topology for $X$ (and the pair $(X, \tau)$ a fuzzy topological space):

(i) $\quad \tau$ contains every constant fuzzy set (function) in $X$;

(ii) $\bigcup_{i \in I} A_{i} \in \tau$ whenever each $A_{i} \in \tau(i \in I)$; and

(iii) $A \cap B \in \tau$ whenever $A, B \in \tau$.

The elements of $\tau$ and their complements are called open and closed, respectively. A neighborhoods of a fuzzy set $A$ of a fuzzy topological space $X$ is any fuzzy set $B$ for which there is an open fuzzy set $V$ satisfying $A \subset V \subset B$. Any open fuzzy set $V$ that satisfies $A \subset V$ is called an open neighborhood of $A$. A fuzzy set $A$ in $(X, \tau)$ is called fuzzy compact if and only if open covering of $A$ has a finite subcovering. Similarly, we can define fuzzy Hausdorff spaces.

A net $\left(x_{\lambda}\right)_{\lambda \in \Lambda}$ in a fuzzy topological space $(X, \tau)$ converges to a point $x$ (denoted by $\left.x_{\lambda} \rightarrow x\right)$ : if given a neighborhood $V$ of $x$, there exists a $\lambda_{0} \in \Lambda$ such that $x_{\lambda} \in V$ whenever $\lambda \geq \lambda_{0}$. A point $x$ belongs to the closure of a fuzzy subset $C$ of $X$ if there is a net in $C$ converging to $x$. In general, a net in a fuzzy topological space may converge to several points but in a fuzzy Hausdorff space, the convergence is unique.

A single-valued map $f$ from a fuzzy topological space $X$ to a fuzzy topological space $Y$ is called continuous at some $x \in X$ if $f^{-1}(V)$ is a neighborhood of $x$ or each neighborhood $V$ of $f(x)$. (Here $f^{-1}(V)$ is the fuzzy set in $X$ defined by $\left[\left(f^{-1}(V)\right)(x)=V(f(x))\right]$. For further details, we refer to [8, 10, 16-18, 25-27].

\section{Fuzzy Multifunctions}

A fuzzy multifunction $f$ from a set $X$ into a set $Y$ assigns to each $x$ in $X$, a fuzzy subset $f(x)$ of $Y$. We denote this assignment by $f: X \rightarrow Y$. We can identify $f$ with a fuzzy subset $G_{f}$ of $X \times Y$ and $[f(x)](y)=G_{f}(x, y)$.

If $A$ is a fuzzy subset of $X$, then the fuzzy set $f(A)$ in $Y$ is defined by

$$
[f(A)](y)=\sup _{x \in X}\left[G_{f}(x, y) \wedge A(x)\right] .
$$

The graph $G_{f}$ of $f$ is the fuzzy subset of $X \times Y$ associated with $f$,

$$
G_{f}=\{(x, y) \in X \times Y:[f(x)](y) \neq 0\} .
$$

Definition 3.1: The upper inverse $f^{u}$ of a fuzzy multifunction $f: X \rightarrow Y$, is defined by

$$
\left[f^{u}(A)\right](x)=\inf _{y \in Y}\left[\left(1-G_{f}(x, y)\right) \vee A(y)\right]
$$


Definition 3.2: The lower inverse $f^{\ell}$ of a fuzzy multifunction $f: X \rightarrow Y$ is defined by

$$
\left[f^{\ell}(A)\right](x)=\sum_{y \in Y}\left[G_{f}(x, y) \wedge A(y)\right]
$$

Definition 3.3: The fuzzy multifunction $f: X \rightarrow Y$ is fuzzy closed valued if $f(x)$ is a closed fuzzy set for each $x$. The terms fuzzy open valued and fuzzy compact valued are defined similarly.

Definition 3.4: A fuzzy multifunction $f: X \rightarrow Y$ between two fuzzy topological spaces $X$ and $Y$ is:

(a) upper hemicontinuous at the point $x$, if for every open neighborhood $U$ of $f(x), f^{u}(U)$ is a neighborhood of $x$ in $X$. The fuzzy multifunction $f$ is upper hemicontinuous on $X$ if it is upper hemicontinuous at every point of $X$;

(b) lower hemicontinuous at $x$, if for every open fuzzy set $U$ which intersects $f(x), f^{\ell}(U)$ is a neighborhood of $x$. As above, $f$ is lower hemicontinuous on $X$ if it is lower hemicontinuous at each point of $X$;

(c) continuous if it is both upper and lower hemicontinuous.

For a more detailed account of the concepts outlined above, the reader is referred to Beg $[5,6]$ and Tsiporkova-Hristoskova, De Baets and Kerre [23, 24].

\section{Fuzzy Topological Vector Spaces}

Let $E$ be a vector space over $K$, where $K$ denotes either the real or the complex numbers. Let $A_{1}, A_{2}, \ldots, A_{n}$ be fuzzy subsets of $E$, with $A_{1} \times A_{2} \times A_{3} \times \ldots \times A_{n}$ denoting the fuzzy subset $A$ in $E^{n}$ defined by

$$
A\left(x_{1}, x_{2}, \ldots, x_{n}\right)=\min \left\{A_{1}\left(x_{1}\right), A_{2}\left(x_{2}\right), \ldots, A_{n}\left(x_{n}\right)\right\} .
$$

If $f: E^{n} \rightarrow E$ is defined by $f\left(x_{1}, x_{2} ; \ldots x_{n}\right)=x_{1}+x_{2}+\ldots+x_{n}$, then the fuzzy set $f(A)$ in $E$ is called the sum of the fuzzy sets $A_{1}, A_{2}, \ldots, A_{n}$, and it is denoted by $A_{1}+$ $A_{2} \ldots+A_{n}$. For a fuzzy subset $A$ of $E$ and $t$ a scalar, we denote $t A$ as the image of $A$ under the map $g: E \rightarrow E, g(x)=t x$. If $\alpha$ is a fuzzy set in $K$ and $A$ a fuzzy set in $E$, then the image in $E$ of the fuzzy set $\alpha \times A$, a fuzzy subset of $K \times E[(\alpha \times A)(t, x)=$ $\min \{\alpha(t), A(x)\}]$ under the map $h: K \times E \rightarrow E, h(t, x)=t x$, is denoted by $\alpha A$. A fuzzy set $A$ in $E$ is called convex if for each $t \in[0,1],[t A+(1-t) A](x) \leq A(x)$. The convex hull of a fuzzy set $B$ is the smallest convex fuzzy set containing $B$, and is denoted by $c_{0}(B)$.

Given a topological space $(X, \tau)$, the collection $w(\tau)$ of all fuzzy sets in $X$, which are lower semicontinuous, as a function from $X$ to $[0,1]$ equipped with the usual topology, is a fuzzy topology of $X$. The fuzzy topology $w(\tau)$ is called the fuzzy topology generated by the usual topology $\tau$. The fuzzy usual topology on $K$ is the fuzzy topology generated by the topology of $K$.

Definition 4.1: A fuzzy linear topology on a vector space $E$ over $K$ is a fuzzy topology $\tau$ on $E$ such that the two mappings:

$$
f: E \times E \rightarrow E, \quad f(x, y)=x+y, \text { and }
$$




$$
h: K \times E \rightarrow E, h(t, x)=t x,
$$

are continuous when $K$ has the usual fuzzy topology, with $K \times E, E \times E$ being the corresponding product fuzzy topologies.

A linear space with a fuzzy linear topology is called a fuzzy topological vector space.

Lemma 4.2: In a fuzzy topological vector space $X$, the algebraic sum of a compact fuzzy set and a closed fuzzy set is closed fuzzy set.

Proof: Let $A$ be a compact fuzzy subset and $B$ be a closed fuzzy subset of $X$. Let a net $\left\{x_{\lambda}+y_{\lambda}\right\}$ in $A+B$ satisfy $x_{\lambda}+y_{\lambda} \rightarrow z$. Since $A$ is compact fuzzy set, we can assume (by passing to a subnet) that $x_{\lambda} \rightarrow x \in A$. The continuity of the algebraic operations imply:

$$
y_{\lambda}=\left(x_{\lambda}+y_{\lambda}\right)-x_{\lambda} \rightarrow z-x=y
$$

Since $B$ is a closed fuzzy subset, therefore, $y \in B$. So $z=x+y \in A+B$. Hence $A+B$ is a close fuzzy set.

Lemma 4.3: In a fuzzy topological vector space $X$, the algebraic sum of two compact fuzzy sets is a compact fuzzy set.

Proof: Similar to Lemma 4.2 .

Theorem 4.4: Let $K$ be a compact fuzzy subset of a fuzzy topological vector space $X$. Suppose $K \subset U$, where $U$ is an open fuzzy subset. Then there is a neighborhood $W$ of origin such that $K+W \subset U$.

Proof: For each $x \in K$, there is a neighborhood $V_{x}$ of origin such that $x+V_{x} \subset U$. Choose an open neighborhood $W_{x}$ of origin so that $W_{x}+W_{x} \subset V_{x}$ for each $x$. Since $K$ is a compact fuzzy set, there is a finite set $\left\{x_{1}, x_{2}, \ldots, x_{n}\right\}$ of points with $K \subseteq \bigcup_{i=1}^{n}\left(x_{i}+W_{x_{i}}\right)$. Set $W=\bigcap_{i=1}^{n} W_{x_{i}}$. For every $x \in K$, there is some $x_{i}$ satisfying $x \in x_{i}+W_{x_{i}}$ For this $x_{i}$,

$$
\begin{gathered}
x+W=\left(x-x_{i}\right)+\left(x_{i}+W\right) \subset W_{x_{i}}+x_{i}+W_{x_{i}} \\
\subset x_{i}+V_{x_{i}} \subset U .
\end{gathered}
$$

Hence $K+W \subset U$.

Theorem 4.5: Let $X$ be a fuzzy topological vector space. If each $A_{i}(i=1,2, \ldots, n)$ is compact, then $c_{0}\left(\bigcup_{i=1}^{n} A_{i}\right)$ is a compact fuzzy set.

Proof: Since the continuous image of a compact fuzzy set is a compact fuzzy set and the

$$
c_{0}\left(\bigcup_{i=1}^{n} A_{i}\right)=f\left(K \times A_{1} \times A_{2} \times \ldots \times A_{n}\right) .
$$

Hence $C_{0}\left(\bigcup_{i=1}^{n} A_{i}\right)$ is a compact fuzzy set.

Definition 4.6: A fuzzy topological vector space $E$ is called locally convex if it has a base at the origin of convex fuzzy sets.

For basic concepts and details regarding fuzzy topological vector spaces, we refer to $[12-14,17,18]$. 


\section{Vector-Valued Fuzzy Multifunctions}

When the range space of a fuzzy multifunction is a vector space, then there are additional natural operations on fuzzy multifunctions.

Definition 5.1: If $f, g: X \rightarrow Y$ are two fuzzy multifunctions, where $Y$ is a vector space, then we define:

1. The sum fuzzy multifunction $f+g$ by

$$
(f+g)(x)=f(x)+g(x)=\{y+z: y \in f(x) \text { and } z \in g(x)\} .
$$

2. The convex hull fuzzy multifunction $c_{0}(f)$ of $f$ by

$$
\left(c_{0}(f)\right)(x)=c_{0}(f(x)) \text {. }
$$

3. If $Y$ is a fuzzy topological vector space, the closed convex hull fuzzy multifunction $c \ell\left(c_{0}(f)\right)$ of $f$ by

$$
\left(c \ell\left(c_{0}(f)\right)\right)(x)=c \ell\left(c_{0}(f(x))\right)
$$

Lemmas 4.2 and 4.3 imply the following theorem.

Theorem 5.2: Let $f, g: X \rightarrow Y$ be two fuzzy multifunctions from a fuzzy topological space $X$ into a fuzzy topological vector space $Y$ :

1. If $f$ is closed valued and $g$ is compact-valued, then $f+g$ is closed valued.

2. If $f$ and $g$ are compact valued, then $f+g$ is compact valued.

Theorem 5.3: Let $f, g: X \rightarrow Y$ be two fuzzy multifunctions from a fuzzy topological space $X$ into a fuzzy topological vector space $Y$. If $f$ and $g$ are compact valued and upper hemicontinuous at a point $x_{0}$, then $f+g$ is upper hemicontinuous at $x_{0}$.

Proof: Let $f$ and $g$ be upper hemicontinuous fuzzy multifunctions at the point $x_{0}$. Suppose $f\left(x_{0}\right)+g\left(x_{0}\right) \subset G$, where $G$ is an open fuzzy subset of $Y$. By Theorem 4.4, there is a neighborhood $V$ of origin such that $f\left(x_{0}\right)+g\left(x_{0}\right)+V \subset G$. Select an open neighborhood $W$ of origin with $W+W \subset V$. Since $f\left(x_{0}\right) \subset f\left(x_{0}\right)+W$ and $f\left(x_{0}\right)+W$ is open, the upper hemicontinuity of $f$ at $x_{0}$ guarantees the existence of an open neighborhood $N_{1}$ of $x_{0}$ such that $f\left(N_{1}\right) \subset f\left(x_{0}\right)+W$. Similarly, there exists an open neighborhood $N_{2}$ of $x_{0}$ with $g\left(N_{2}\right) \subset g\left(x_{0}+W\right.$. Let $N=N_{1} \cap N_{2}$, then $N$ is an open neighborhood of $x_{0}$ and

$$
(f+g)(N) \subset f\left(N_{1}\right)+g\left(N_{2}\right) \subset f\left(x_{0}\right)+W+g\left(x_{0}\right)+W \subset G .
$$

It further implies that $f+g$ is upper hemicontinuous fuzzy multifunctions at $x_{0}$.

Theorem 5.4: Let $f, g: X \rightarrow Y$ be two fuzzy multifunctions from a fuzzy topological space $X$ into a fuzzy topological vector space $Y$. If $f$ and $g$ are also lower hemicontinuous fuzzy multifunctions at a point $x_{0}$, then $f+g$ is also lower hemicontinuous fuzzy multifunctions at $x_{0}$.

Proof: Suppose $\left[f\left(x_{0}\right)+g\left(x_{0}\right)\right] \cap U \neq \phi$, where $U$ is open fuzzy subset. Then there are $y$ in $f\left(x_{0}\right)$ and $z \in g\left(x_{0}\right)$, with $y+z \in U$. Thus, there is an open neighborhood $V$ of origin such that $y+V+z+V \subset U$. Since $y \in f\left(x_{0}\right) \cap(y+V)$ and $f$ is lower hemicontinuous fuzzy multifunction at $x_{0}, f^{\ell}(y+V)$ is a neighborhood of $x_{0}$.

Similarly, $g^{\ell}(z+V)$ is a neighborhood of $x_{0}$. Hence, if $x \in f^{\ell}(y+V) \cap g^{\ell}(z+V)$, then $[f(z)+g(x)] \cap U \neq \phi$. 
Theorem 5.5: Let $f_{i}: X \rightarrow Y(i=1,2, \ldots, n)$ be (single-valued) fuzzy functions from a fuzzy topological space $X$ into a fuzzy topological vector space $Y$, and the fuzzy multifunction $f: X \rightarrow Y$ be given by $f(x)=\left\{f_{1}(x), f_{2}(x), \ldots, f_{n}(x)\right\}$. If each $f_{i}$ is continuous at a point $x_{0} \in X$, then the fuzzy multifunction $f$ is continuous at $x_{0}$.

Proof: Suppose $f\left(x_{0}\right)=\left\{f_{1}\left(x_{0}, f_{2}\left(x_{0}\right), \ldots, f_{n}\left(x_{0}\right)\right\} \subset U\right.$, where $U$ is an open fuzzy subset of $Y$. Then:

$$
V=\bigcap_{i=1}^{n} f_{i}^{-1}(U)
$$

is an open neighborhood of $x_{0}$ such that $x \in V$ implies $f(x) \subset U$. It further implies that the fuzzy multifunction $f$ is upper hemicontinuous.

Next, suppose $f\left(x_{0}\right) \cap W \neq \phi$ for some open fuzzy subset $W$ of $Y$. If $f_{n}\left(x_{0}\right) \in W$, then $P=f_{n}^{-1}(W)$ is a neighborhood of $x_{0}$, and $x \in P$ implies $f(P) \cap W \neq \phi$. It further implies that the fuzzy multifunction $f$ is lower semicontinuous. Hence the fuzzy multifunction $f$ is continuous.

Theorem 5.6: Let $f_{i}: X \rightarrow Y(i=1,2,3, \ldots, n)$ be (single-valued) fuzzy functions from a fuzzy topological space $X$ into a locally convex fuzzy topological vector space $Y$, and $f: X \rightarrow Y$ be given by $f(x)=\left\{f_{1}(x), f_{2}(x), \ldots, f_{n}(x)\right\}$. If each $f_{i}$ is continuous at some point $x_{0}$, then the convex hull fuzzy multifunction $c_{0}(f)$ is continuous at $x_{0}$.

Proof: Suppose that $\left(c_{0}(f)\right)(x) \subset U$, where $U$ is an open fuzzy subset of the locally convex fuzzy topological vector space $Y$. By Theorem 4.5, $\left(c_{0}(f)\right)(x)$ is compact. Theorem 4.4 further implies that there exists an open convex neighborhood $W$ of origin satisfying $\left(c_{0}(f)\right)\left(x_{0}\right)+W \subset U$. From $f\left(x_{0}\right) \subset f\left(x_{0}\right)+W$ and the upper hemicontinuity of $f$ at $x_{0}$ (Theorem 5.5), there exists a neighborhood $V$ of $x_{0}$ such that $f(x) \subset f\left(x_{0}\right)+W$ for each $x \in V$. So, if $x \in V$, then $\left(c_{0}(f)\right)(x) \subset\left(c_{0}(f)\right)\left(x_{0}\right)+$ $W \subset U$. This also implies that $c_{0}(f)$ is an upper hemicontinuous fuzzy multifunction at $x_{0}$.

Next, let $\left(c_{0}(f)\right)\left(x_{0}\right) \cap U \neq \phi$ for some open fuzzy subset $U$. Pick $\lambda_{i} \geq 0$ $(i=1,2, \ldots, n)$, with

$$
\sum_{i=1}^{n} \lambda_{i}=1 \text { and } \sum_{i=1}^{n} \lambda_{i} f_{i}\left(x_{0}\right) \in U
$$

The fuzzy function $g: X \rightarrow Y$ defined by $g(x)=\sum_{i=1}^{n} \lambda_{i} f_{i}(x)$ is continuous at $x_{0}$ (by definition of fuzzy topological vector spaces). This implies that there exists a neighborhood $V$ of $x_{0}$ such that $x \in V$ implies $\sum_{i=1}^{n} \lambda_{i} f_{i}(x) \in U$.

Therefore, $\left(\left(c_{0}\right)(f)\right)(x) \cap U \neq \phi$ for each $x \in V$. Thus $c_{0}(f)$ is a lower hemicontinuous fuzzy multifunction at $x_{0}$. Hence, $c_{0}(f)$ is a continuous fuzzy multifunction at $x_{0}$.

Theorem 5.7: Let $X$ be a fuzzy topological space and $Y$ be a locally convex fuzzy topological vector space. Let $f: X \rightarrow Y$ be an upper hemicontinuous fuzzy multifunction at $x$. If $\left(c \ell\left(c_{0}(f)\right)\right)(x)$ is compact, then $c \ell\left(c_{0}(f)\right)$ is an upper hemicontinuous fuzzy multifunction at $x$.

Proof: Let $\left(c \ell\left(c_{0}(f)\right)\right)(x) \subset P$ for some open fuzzy set $P$. If $\left(c \ell\left(c_{0}(f)\right)\right)(x)$ is compact, then there is a convex neighborhood $V$ of origin with $\left(c \ell\left(c_{0}(f)\right)\right)(x)+V+$ $V \subset P$ (by definition of local convexity and Theorem 4.4). Lemma 4.2 further implies that $\left(c \ell\left(c_{0}(f)\right)\right)(x)+c \ell(V)$ is a closed convex fuzzy set. Since $f$ is an upper hemicontinuous fuzzy multifunction at $x, f^{u}(f(x)+V)$ is a neighborhood of $x$. If $z \in f^{u}(f(x)+V)$, then $f(z) \subset f(x)+V$, so 


$$
\begin{gathered}
\left(c \ell\left(c_{0}(f)\right)\right)(z) \subset\left(c \ell\left(c_{0}(f)\right)\right)(x)+c \ell(V) \\
\subset\left(c \ell\left(c_{0}(f)\right)\right)(x)+V+V \subset P .
\end{gathered}
$$

Therefore, $\left(c \ell\left(c_{0}(f)\right)\right)^{u}(P)$ includes $f^{u}(f(x)+V)$. Hence $c \ell\left(c_{0}(f)\right)$, is an upper hemicontinuous fuzzy multifunction at $x$.

Definition 5.8: A fuzzy multifunction $f: X \rightarrow Y$ from a fuzzy topological space $X$ into a fuzzy topological vector space $Y$ is upper demicontinuous if $f^{u}(\{y \in Y$ : $h(y)<\alpha$ where $h$ is a continuous linear fuzzy single valued function from $Y$ into $K\}$ ) is an open subset of $X$.

Theorem 5.9: A compact valued fuzzy multifunction $f: X \rightarrow Y$ from a fuzzy topological space $X$ into a fuzzy topological vector space $Y$ is upper demicontinuous if and only if $\mathrm{cl}\left(c_{0}(f)\right)$ is upper demicontinuous.

Proof: Let $H \subset Y$ be an open half fuzzy space of the form $H=\{y \in Y: \ell(y)<\alpha\}$, where $\ell: Y \rightarrow K$ is a continuous linear fuzzy single valued function. Since $\ell$ is a linear and continuous fuzzy function,

$$
\max \{\ell(y): y \in f(x)\}=\max \left\{\ell(y): y \in\left(c \ell\left(c_{0}(f)\right)\right)(x)\right\}
$$

It implies $f(x) \subset H$ if and only if $\left(c \ell\left(c_{0}(f)\right)\right)(x) \subset H$. Hence $f$ is upper demicontinuous if and only if $c \ell\left(c_{0}(f)\right)$ is also demicontinuous.

\section{References}

[1] Albrycht, J. and Maltoka, M., On fuzzy multivalued functions, Fuzzy Sets and Systems 12 (1984), 61-69.

[2] Aubin, J.P. and Ekeland, I., Applied Nonlinear Analysis, Wiley-Interscience 1984.

[3] Aubin, J.P. and Frankowska, H., Set-Valued Analysis, Birkhauser, Boston 1990.

[4] Beg, I., Fixed points of fuzzy multivalued mappings with values in fuzzy ordered sets, J. Fuzzy Math. 6:1 (1998), 127-131.

[5] Beg, I., Continuity of fuzzy multifunctions, J. Appl. Math. and Stoch. Anal. 12:1 (1999), 17-22.

[6] Beg, I., Fuzzy closed graph fuzzy multifunctions, Fuzzy Sets E Systems 115:3 (2000), 451-454.

[7] Beg, I., Operations on fuzzy multifunctions and fuzzy maximum principle, submitted.

[8] Berge, C., Topological Spaces, MacMillan, New York 1963. English trans. by E.M. Patterson of Espaces Topologiques Fonctions Multivoques, Dunod, Paris 1959.

[9] Butanriu, D., Fixed points for fuzzy mappings, Fuzzy Sets and Systems 7 (1982), 191-207.

[10] Chang, C.L., Fuzzy topological spaces, J. Math. Anal. Appl. 24 (1968), 182-190.

[11] Heilpern, S., Fuzzy mappings and fixed point theorem, J. Math. Anal. Appl. 83 (1981), 566-569.

[12] Katsaras, A.K., Fuzzy topological vector spaces I, Fuzzy Sets and Systems 6 (1981), 85-95.

[13] Katsaras, A.K., Fuzzy topological vector spaces II, Fuzzy Sets and Systems 12 
(1984), 143-154.

[14] Katsaras, A.K. and Liu, D.B., Fuzzy vector spaces and fuzzy topological vector spaces, J. Math. Anal. Appl. 58 (1977), 135-146.

[15] Klein, E. and Thompson, A.C., Theory of Correspondences: Including Applications to Mathematical Economics, John Wiley and Sons 1984.

[16] Li, H.X. and Yen, V.C., Fuzzy Sets and Fuzzy Decision Making, CRC Press, London 1995.

[17] Lowen, R., Fuzzy topological spaces and fuzzy compactness, J. Math. Anal. Appl. 56 (1976), 621-633.

[18] Lowen, R., A comparison of different compactness notions in fuzzy topological spaces, J. Math. Anal. Appl. 64 (1978), 445-454.

[19] Ozbakir, O.B. and Aslim, G., On some types of fuzzy continuous multifunctions, Bull. Cal. Math. Soc. 88 (1996), 217-230.

[20] Papageorgiou, N.S., Fuzzy topology and fuzzy multifunctions, J. Math. Anal. Appl. 109 (1985), 397-425.

[21] Pu, P. and Liu, Y., Fuzzy topology I: Neighborhood structure of a fuzzy point and Moore-Smith convergence, J. Math. Anal. Appl. 76 (1980), 571-599.

[22] Tsiporkova-Hristoskova, E., De Baets, B. and Kerre, E., A detailed study of direct and inverse images under fuzzy multivalued mappings, J. Fuzzy Math 3 (1995), 191-208.

[23] Tsiporkova-Hristoskova, E., De Baets, B. and Kerre, E., A fuzzy inclusion based approach to upper inverse images under fuzzy multivalued mappings, Fuzzy Sets and Systems 85 (1997), 93-108.

[24] Tsiporkova-Hristoskova, E., De Baets, B. and Kerre, E., Continuity of fuzzy multivalued mappings, Fuzzy Sets and Systems 94 (1998), 335-348.

[25] Weiss, M.D., Fixed points, separation and induced topologies for fuzzy sets, J. Math. Anal. Appl. 50 (1972), 142-150.

[26] Zadah, L.A., Fuzzy sets, Inform. Control. 8 (1965), 338-353.

[27] Zimmermann, H.J., Fuzzy Set Theory and its Applications, Kluwer Academic Publishers, Boston 1991. 


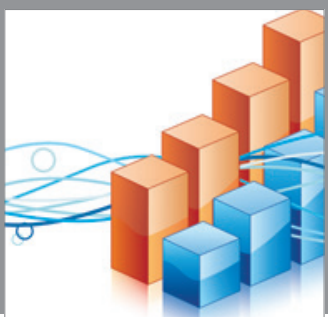

Advances in

Operations Research

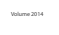

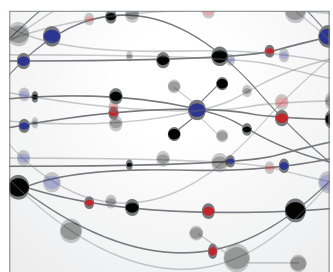

\section{The Scientific} World Journal
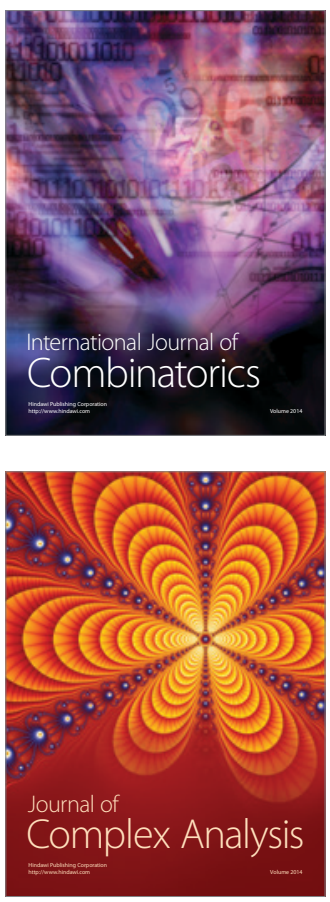

International Journal of

Mathematics and

Mathematical

Sciences
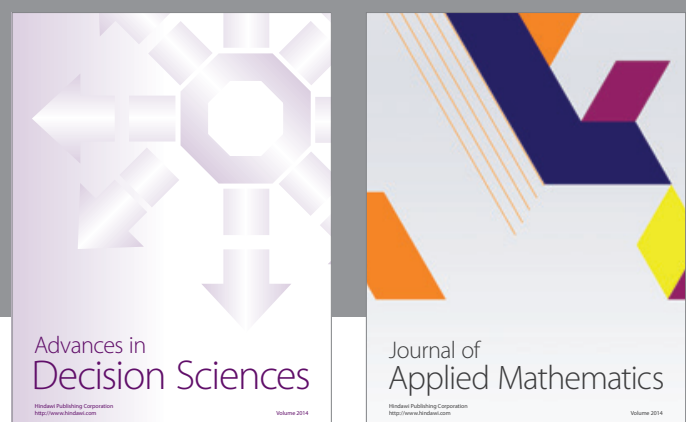

Journal of

Applied Mathematics
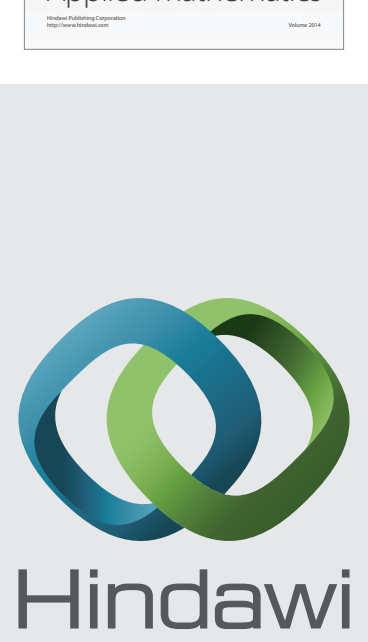

Submit your manuscripts at http://www.hindawi.com
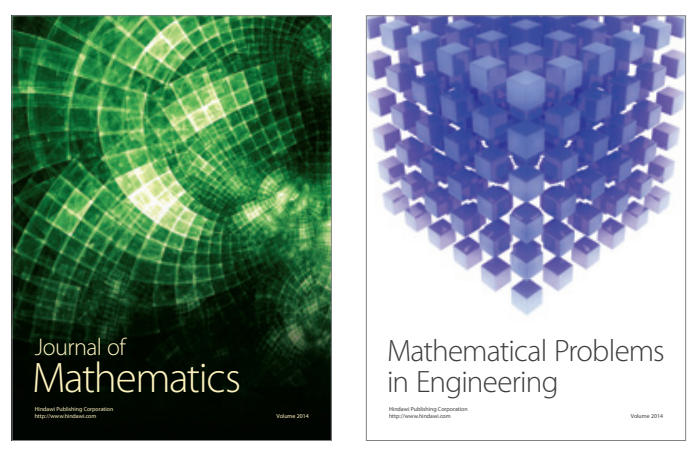

Mathematical Problems in Engineering
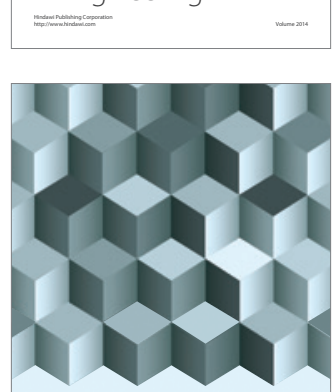

Journal of

Function Spaces
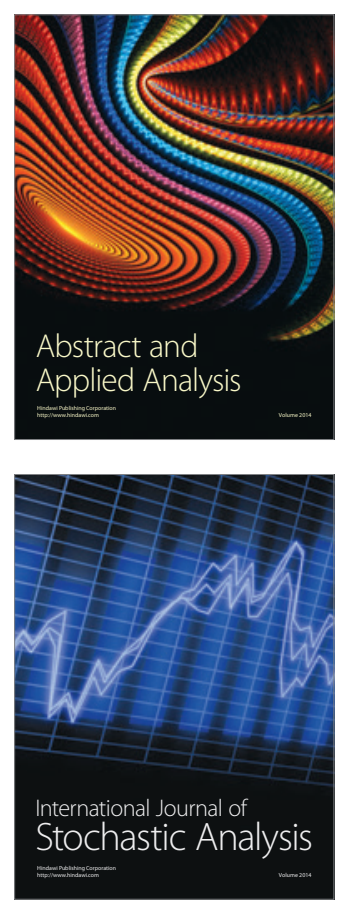

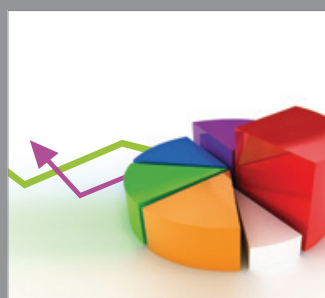

ournal of

Probability and Statistics

Promensencen
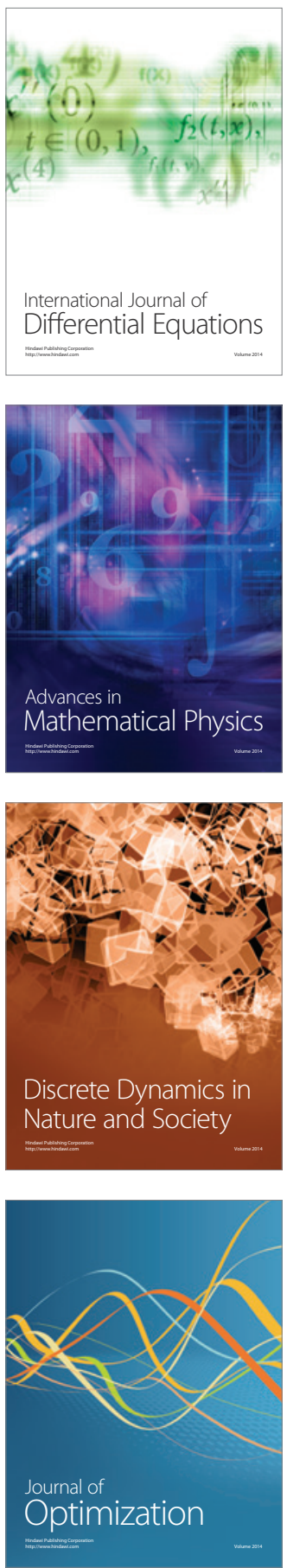